\title{
Irregularities in the distribution of primes in an arithmetic progression
}

\author{
by
}

Ti ZuO XUAN (Beijing)

1. Introduction. For $x \geq 2$ real, and $q$ and $a$ coprime positive integers, set

$$
\theta(x ; q, a)=\sum_{\substack{p \leq x \\ p \equiv a(\bmod q)}} \log p=\frac{x}{\varphi(q)}(1+\Delta(x ; q, a)),
$$

where $\varphi$ is Euler's function.

The prime number theorem for arithmetic progressions is equivalent to the statement that $\Delta(x ; q, a)=o(1)$ as $x \rightarrow \infty$, for fixed $q$ and $a$. The Siegel-Walfisz theorem gave a uniform upper estimate for the function $\Delta$, and the Bombieri-Vinogradov theorem gave a mean value estimate for $\Delta$.

Montgomery conjectured that if $(a, q)=1$ then

$$
|\Delta(x ; q, a)| \ll_{\varepsilon}(q / x)^{1 / 2-\varepsilon} \log x
$$

uniformly for $q \leq x$, for any given $\varepsilon>0$.

Recently, Friedlander and Granville [1] disproved Montgomery's conjecture (1). They showed that for any $A>0$ there exist arbitrarily large values of $x$ and integers $q \leq x /(\log x)^{A}$ and $a$ with $(a, q)=1$ for which $|\Delta(x ; q, a)| \gg 1$.

Then Friedlander, Granville, Hildebrand and Maier [2] further showed that (1) fails to hold for almost all moduli $q$ as small as $x \exp \left\{-(\log x)^{1 / 3-\delta}\right\}$, for any fixed $\delta>0$, if the parameter $\varepsilon$ in (1) is sufficiently small.

They also showed the following

TheOrem A [2]. Let $\varepsilon>0$. There exist $N(\varepsilon)>0$ and $q_{0}=q_{0}(\varepsilon)>0$ such that for any $q>q_{0}$ and any $x$ with

$$
q(\log q)^{N(\varepsilon)}<x \leq q \exp \left\{(\log q)^{1 / 3}\right\},
$$

Project supported by the National Natural Science Foundation of the People's Republic of China. 
there exist numbers $x_{ \pm}$with $x / 2<x_{ \pm} \leq 2 x$ and integers $a_{ \pm}$coprime to $q$ such that

$$
\begin{aligned}
& \Delta\left(x_{+} ; q, a_{+}\right) \geq(\log x)^{-5} y^{-(1+\varepsilon) \delta_{1}(x, y)}, \\
& \Delta\left(x_{-} ; q, a_{-}\right) \leq-(\log x)^{-5} y^{-(1+\varepsilon) \delta_{1}(x, y)},
\end{aligned}
$$

where $y=x / q$ and $\delta_{1}(x, y)=3 \log \left(\log y / \log _{2} x\right) / \log (\log x \log y)$. (Here $\log _{2} x=\log \log x$.)

It follows from Theorem A that (1) fails to hold for all moduli $q$ with

$$
x /(\log x)^{N(\varepsilon)} \geq q>x \exp \left\{-(\log x)^{1 / 5-\delta}\right\} .
$$

In this note, our purpose is to extend the above result by showing the following

TheOREM. For $\varepsilon>0$, there exists $q_{0}(\varepsilon)>0$ such that for any $q>q_{0}(\varepsilon)$ and any $x$ with

$$
q(\log q)^{1+\varepsilon}<x \leq q \exp \left\{(\log q)^{1 / 3}\right\},
$$

there exist numbers $x_{ \pm}$with $x / 2<x_{ \pm} \leq 2 x$ and integers $a_{ \pm}$coprime to $q$ such that

$$
\begin{aligned}
& \Delta\left(x_{+} ; q, a_{+}\right) \geq(\log x)^{-3} y^{-(1+\varepsilon) \delta_{2}(x, y)}, \\
& \Delta\left(x_{-} ; q, a_{-}\right) \leq-(\log x)^{-3} y^{-(1+\varepsilon) \delta_{2}(x, y)},
\end{aligned}
$$

where $y=x / q$ and $\delta_{2}(x, y)=2 \log _{2} y / \log _{2} x$.

It follows from the Theorem that (1) fails to hold for all moduli $q$ with

$$
x /(\log x)^{6+\varepsilon} \geq q>x \exp \left\{-(\log x)^{1 / 4-\delta}\right\} .
$$

The exponent $1 / 4$ is the best possible, using this method.

Moreover, we note that the estimates (5) and (6) are slightly better than (2) and (3) for $q<x \exp \left\{-\left(\log _{2} x\right)^{4}\right\}$.

2. Some lemmas. The following two lemmas are Theorem B2 and Proposition 11.1 of [2], respectively.

Lemma 1 [2]. For $z \geq z_{0}, h \leq z / 2, k \geq 1$, and $P$ the product of any $k$ primes all of which are in the interval $(z-h, z]$, we have

$$
(-1)^{j-1} r_{P}(y):=(-1)^{j-1}\left(\sum_{n \leq y,(n, P)=1} 1-\frac{\varphi(P)}{P} y\right) \geq \frac{1}{4} y\left(\begin{array}{l}
k \\
j
\end{array}\right) z^{-j},
$$

for every integer $j$ with $1 \leq j \leq k / 5$ and every real $y$ with $(z-h)^{j} \geq y \geq$ $4 j z^{j} /(k-j+1)$.

Lemma 2 [2]. Fix $\varepsilon>0$. For any squarefree integer $n>1$ all of whose prime factors are $\leq n^{1-\varepsilon}$, there exists a divisor $P$ of $n$, with $n / P$ prime, 
such that if $(a, P)=1, x \geq P^{2}$, and $x \geq h \geq x \exp (-\sqrt{\log x})$, then

$$
\theta(x+h ; P, a)-\theta(x ; P, a)=\frac{h}{\varphi(P)}\left(1+O\left(e^{-c \log x / \log P}+e^{-c \sqrt{\log x}}\right)\right),
$$

where $c$ is a constant depending only on $\varepsilon$.

3. Proof of Theorem. For the proof of this result we use combinatorial means. This is a simple modification of the argument in [2]. We only prove (5), the proof of (6) is similar.

Let $y=x / q$. Define $v$ to be the positive solution of the equation

$$
\left(\lambda v \log _{2} x \cdot \log x / \log y\right)^{v}=y,
$$

where $\lambda=1+N / \log y, 1 \leq N \leq 9 \log y$, and the positive integer $N$ will be given in the latter part of the proof.

We pick $j=[v]-1$ or $j=[v]$ so that $j$ is odd. Then we take

$$
l=y^{1 / j}(\log y / \log x),
$$

and

$$
z=(l+1 / 2) \log x / \log y, \quad h=(1 / 2) \log x / \log y,
$$

so that $(z-h)^{j}=y$. By the definition of $v$, we have $v \leq \log y$ and

$$
v \geq\left(\log y / \log _{2} x\right)\left(1+O\left(\log _{3} x / \log _{2} x\right)\right) .
$$

From this and the definition of $v$, we deduce

$$
v \leq \log y / \log _{2} x .
$$

Using the estimates (10) and (11), we obtain

(12) $\lambda \log y\left(1+O\left(\log _{3} x / \log _{2} x\right)\right) \leq l \leq \lambda \log y \exp \left\{(5 / 2) \log _{2}^{2} x / \log y\right\}$.

Now take $k=1+\left[c \log x /\left(20 j \log _{2}^{2} x\right)\right]$, where $c$ is the constant $c$ of Lemma 2 . From this, the definition of $j,(10),(11)$ and the first inequality of (12), we deduce

$$
(z-j)^{j}=y \geq 4 j z^{j} /(k-j+1) .
$$

Let $n$ be the product of any $k+1$ primes in $(z-h, z]$ that do not divide q. By Huxley's theorem (cf. [2]) we have $\pi(z)-\pi(z-h) \sim h / \log z$ as $z \rightarrow \infty$. Now we choose $N$ in (7). First we note that the number of distinct prime factors of $q$ does not exceed $(1+\varepsilon) \log x / \log _{2} x$. When $N$ runs over $1,2, \ldots,[9 \log y]$, the intervals $(z-h, z]$ do not overlap. Thus, there is at least one $N$ such that the corresponding interval $(z-h, z]$ contains less than $\nu_{q}=\left[(1+\varepsilon) \log x /\left(8 \log y \cdot \log _{2} x\right)\right]$ primes that divide $q$. By this we see that the interval $(z-h, z]$ contains at least $\nu_{q}+k+1$ primes. Moreover, we choose $P$ as in Lemma 2, with $\varepsilon=1 / 2$. 
As in [2], we consider the matrix $\mathcal{M}=\left(a_{r s}\right)$, where $a_{r s}=\log (r P+q s)$ if $r P+q s$ is prime, and $a_{r s}=0$ otherwise, and where $r$ and $s$ run over the values $R<r \leq 2 R$ and $1 \leq s \leq y$ with

$$
R=(x / P) \exp \{-\sqrt{\log x}\} .
$$

Let $|\mathcal{M}|$ denote the sum of the entries of $\mathcal{M}$. For given $s$, the sum of entries in the sth column equals

$$
\theta(2 R P+q s ; P, q s)-\theta(R P+q s ; P, q s) .
$$

This vanishes if $(q s, P)>1$. Now we consider the case when $s$ satisfies $(q s, P)=1$. Applying Lemma 2 with $x=P R+q s, h=P R, a=q s$ yields

$$
|\mathcal{M}|=\sum_{n \leq y,(n, P)=1} \frac{R P}{\varphi(P)}\left(1+O\left(y^{-3}\right)\right),
$$

where we have used the inequalities

$$
c \log x / \log P \geq c \log x /(k \log z) \geq 3 \log y,
$$

which follows from (9)-(11) and the second inequality of (12).

By the definition of $r_{P}(y)$, we further have

$$
|\mathcal{M}|=R\left\{y+(P / \varphi(P)) r_{P}(y)\right\}\left(1+O\left(y^{-3}\right)\right) .
$$

On the other hand, the number of $r$ satisfying $R<r \leq 2 R$ and $(r, q)=1$ equals

$$
R \varphi(q) / q+O(\tau(q))=R \varphi(q) / q\left(1+O\left(y^{-3}\right)\right) .
$$

Therefore we may choose some such row (say row $r_{0}$ ) such that the sum of the entries in this row is more than

$$
(q / \varphi(q))\left\{y+(P / \varphi(P)) r_{P}(y)\right\}\left(1+O\left(y^{-3}\right)\right) .
$$

Let $x_{0}=x_{+}=r_{0} P+q y$ and $a=a_{+}=r_{0} P$, so $(a, q)=1$. Now, the sum of the entries in row $r_{0}$ equals

$$
\theta\left(r_{0} P+q y ; q, r_{0} P\right)-\theta\left(r_{0} P ; q, r_{0} P\right)=\theta\left(x_{0} ; q, a\right) .
$$

(Since, by (14), $r_{0} P \leq 2 R P<q$, we have therefore $\theta\left(r_{0} P ; q, r_{0} P\right)=0$.) By the definitions of $\theta$ and $\Delta$ and (14) we obtain

$$
\theta\left(x_{0} ; q, a\right)=(q y / \varphi(q))\left(1+\Delta\left(x_{0} ; q, a\right)\right)\left(1+O\left(y^{-3}\right)\right) .
$$

Combining (16) and (17) yields

$$
(-1)^{j-1} \Delta\left(x_{0} ; q, a\right) \geq(-1)^{j-1} \frac{P}{\varphi(P)} \cdot \frac{r_{P}(y)}{y}+O\left(y^{-2}\right) .
$$


Thus, by Lemma 1, (9)-(11) and the second inequality of (12) we obtain

$$
\begin{aligned}
(-1)^{j-1} \frac{r_{P}(y)}{y} & \geq \frac{1}{4}\left(\begin{array}{c}
k \\
j
\end{array}\right) \frac{1}{z^{j}} \gg \frac{1}{\sqrt{j}}\left(\frac{e k}{j z}\right)^{j} \gg\left(\frac{c_{1} \log y}{j^{2} l \log _{2}^{2} x}\right)^{j} \\
& \gg \exp \left\{-(1+\varepsilon) \frac{\log y}{\log _{2} x}\left(2 \log _{2} y+\frac{5 \log _{2}^{2} x}{2 \log y}\right)\right\}
\end{aligned}
$$

(where $c_{1}=c e / 30$ ). From this, the desired estimate (5) follows.

\section{References}

[1] J. Friedlander and A. Granville, Limitations to the equi-distribution of primes I, Ann. of Math. 129 (1989), 363-382.

[2] J. Friedlander, A. Granville, A. Hildebrand and H. Maier, Oscillation theorems for primes in arithmetic progressions, J. Amer. Math. Soc. 4 (1991), 25-86.

Department of Mathematics

Beijing Normal University

Beijing 100875, China 